Original Article

\title{
Effects of brain-computer interface-based functional electrical stimulation on brain activation in stroke patients: a pilot randomized controlled trial
}

\author{
EunJung Chung, PT, PhD ${ }^{1)}$, Jung-Hee Kim, PT, MSc ${ }^{2)}$, Dae-Sung Park, PT, PhD ${ }^{3)}$, \\ Byoung-Hee Lee, PT, PhD $\left.{ }^{4}\right)^{*}$ \\ 1) Department of Physical Therapy, Andong Science College, Republic of Korea \\ 2) Department of Physical Therapy, Sahmyook University, Republic of Korea \\ 3) Department of Physical Therapy, Konyang University, Republic of Korea \\ 4) Department of Physical Therapy, Sahmyook University: 815 Hwarang-ro, Nowon-gu, Seoul 139-742, \\ Republic of Korea
}

\begin{abstract}
Purpose] This study sought to determine the effects of brain-computer interface-based functional electrical stimulation (BCI-FES) on brain activation in patients with stroke. [Subjects] The subjects were randomized to in a BCI-FES group ( $\mathrm{n}=5$ ) and a functional electrical stimulation (FES) group ( $\mathrm{n}=5$ ). [Methods] Patients in the BCI-FES group received ankle dorsiflexion training with FES for 30 minutes per day, 5 times under the brain-computer interface-based program. The FES group received ankle dorsiflexion training with FES for the same amount of time. [Results] The BCI-FES group demonstrated significant differences in the frontopolar regions 1 and 2 attention indexes, and frontopolar 1 activation index. The FES group demonstrated no significant differences. There were significant differences in the frontopolar 1 region activation index between the two groups after the interventions. [Conclusion] The results of this study suggest that BCI-FES training may be more effective in stimulating brain activation than only FES training in patients recovering from stroke.

Key words: Brain-computer interface, Stroke rehabilitation, Brain activation
\end{abstract}

(This article was submitted Aug. 21, 2014, and was accepted Sep. 10, 2014)

\section{INTRODUCTION}

A brain-computer interface (BCI) mesures neuronal signals by using an electrocorticogram (ECoG), electroencephalogram (EEG), event-related brain potentials (ERPs), magnetoencephalography (MEG), or real-time magnetic resonance imaging (Rt-MRI) without peripheral physiologic activities ${ }^{1)}$. Brain activation is recorded through amplification and online classified by an algorithm ${ }^{2}$.

Biofeedback consists of sensory stimulation such as visual, auditory, tactile, and proprioceptive, stimulation sent to direct the movement of muscles by the brain, and functional electrical stimulation (FES) uses electrical stimulation to overcome paralysis by directly activating nerves to stimulate motor function. Feedback varies according to the extent of brain activation ${ }^{1)}$. Most BCIs are designed based on motor imagery (MI) and movement execution $(\mathrm{ME})^{3)}$.

BCI technology is used in the fields of medical treatment and education as a form of neuro-feedback. Early technol-

*Corresponding author. Byoung-Hee Lee (E-mail: 3679@syu. ac.kr)

(C2015 The Society of Physical Therapy Science. Published by IPEC Inc. This is an open-access article distributed under the terms of the Creative Commons Attribution Non-Commercial No Derivatives (by-ncnd) License $<$ http://creativecommons.org/licenses/by-nc-nd/3.0/>. ogy was developed to allow individuals with severe motor disabilities such as amyotrophic lateral sclerosis to communicate and interact with their external environment ${ }^{4}$. Later on, BCI technology was applied to rehabilitation exercises for motor neuron rehabilitation in quadriplegic patients with spinal injury ${ }^{1)}$. However, research on the application of BCI for stroke patients is limited ${ }^{3)}$.

FES is used to facilitate normal movement in patients with paralysis due to upper motor neuron diseases such as stroke, multiple sclerosis, and spinal cord injury ${ }^{5}$. The treatment effects of FES application can occur by peripheral or central mechanisms. Peripheral mechanisms entail FES applied to stimulate the patient's remaining motor units to enhance muscular strength, increase range of motion, and reduce stiffness ${ }^{6}$. Central mechanisms occur by a reorganization of the cortex based on neurophysiological responses to help control movement through neural plasticity in stroke patients ${ }^{7)}$.

Recently, BCI technology has been applied to rehabilitation training using FES, robots, and assistance tools as feedback training ${ }^{3}$. The purpose of this study was to assess the effect of BCI-based FES (BCI-FES) in stroke on brain activation.

\section{SUBJECTS AND METHODS}

Ten subjects from S hospital in Seoul, South Korea, 
diagnosed with chronic hemiparetic stroke, were enrolled in this study. The subjects were provided with a full explanation of the experimental procedure, and provided written consent signifying voluntary participation. The Sahmyook University Human Studies Committee approved this study (SYUIRB2012-010). Patient demographic information is provided in Table 1 . The inclusion criteria were as follows: (1) patients were studied more than six months after clinical diagnosis of ischemic or hemorrhagic hemiparetic stroke, (2) scored more than 24 points on the Mini-Mental State Examination, and (3) were able to walk independently more than $10 \mathrm{~m}$. The exclusion criteria were as follows: (1) severe hemineglect, (2) history or current diagnoses of other neurological diseases or musculoskeletal conditions ${ }^{8)}$, and (3) prior adverse reaction to FES application.

The participants in both the BCI-FES group and FES group participated in the program according to the designated schedule and were randomly allocated to either group; one research assistant participated in training, and two research assistants performed study measurements. Research assistants were educated and trained on the usage of equipment, measurement method, and training programs prior to the study to minimize errors. BCI-FES patients received ankle dorsiflexion training with FES for 30 minutes per day for a total of 5 times under the brain-computer interface based program. FES participants received ankle dorsiflexion training with FES for the same time.

In the BCI-FES-based training, FES was applied to train the participants when they were concentrating on the moving ankle motion on the monitor screen, and brainwave patterns were displayed as the ratio of sensorimotor and MBeta waves to theta waves. The BCI equipment consisted of a monitor screen for the subjects, a brainwave measurement tool (PolyG-I), a laptop to record and process brainwave activity, a USB output board to link the brain waves to FES signals when the activity was high, and FES and EEG sensors to receive brainwave information.

As factors of the external environment such as temperature, place, intensity of illumination, noise, and smell can influence performance and brain activity, participants were provided with private space without external stimuli. To measure brainwave activity, electrodes were attached on the frontopolar $1(\mathrm{Fp} 1)$, and frontopolar $2(\mathrm{Fp} 2)$ regions, with the reference electrode attached behind the right earlobe, and ground electrode attached on the left earlobe. For FES training, an inactive electrode was attached to the proximal tibialis anterior (located $5 \mathrm{~cm}$ inferior to the head of fibula), which is an antagonist of plantar flexor muscles, and an active electrode was attached to the distal tibialis anterior (located on the lateral upper $5 \mathrm{~cm}$ of the fibula). The study participants sat comfortably on chairs with armrests and concentrated to move their ankles by looking at a monitor screen displaying a dorsiflexing ankle. The degree of mental concentration was analyzed using a brainwave concentration index. For measuring brain waves, the concentration index was quantified as in the formula above. In a state of concentration, the theta rhythm decreases, while the SMR $(12-15 \mathrm{~Hz})$ and Mid-Beta rhythms $(16-20 \mathrm{~Hz})$ increase. Increased SMR implies unfocused attention ability, whereas Mid-Beta rhythms connote focused attention and cautious-
Table 1. Characteristics of the participants $(\mathrm{N}=10)$

\begin{tabular}{lcc}
\hline & $\begin{array}{c}\text { BCI-FES group } \\
(\mathrm{n}=5)\end{array}$ & $\begin{array}{c}\text { FES group } \\
(\mathrm{n}=5)\end{array}$ \\
\hline Gender $(\%)$ & $4(80.0) / 1(20.0)$ & $4(80.0) / 1(20.0)$ \\
Male/Female & $43.6(10.9)$ & $50.2(7.1)$ \\
Age $(\mathrm{y})$ & $173.1(10.5)$ & $166.2(9.6)$ \\
Height $(\mathrm{cm})$ & $70.3(14.8)$ & $65.6(5.6)$ \\
Weight $(\mathrm{kg})$ & $16.4(19.2)$ & $8.4(2.2)$ \\
Months after stroke & $2(40.0) / 3(60.0)$ & $2(40.0) / 3(60.0)$ \\
Side of hemiplegia $(\%)$ & & \\
Right/Left & $2(40.0) / 3(60.0)$ & $1(20.0) / 4(80.0)$ \\
Type of stroke (\%) & & \\
Infarction/hemmorrahge & &
\end{tabular}

$\mathrm{n}(\%)$ or mean $(\mathrm{SD})$

ness. Therefore, the concentration index was quantified by a ratio involving SMR, Mid-Beta, and theta waves as expressed by the formula: (SMR + Mid-Beta)/Theta.

Before performing measurements, the experimenter passively modulated the stimulated current intensity of frequency $35 \mathrm{~Hz}$, and pulse with $250 \mu$ s from $1 \mathrm{~mA}$ to 50 $\mathrm{mA}$ according to the response from the subject's ankle joint, and input them as they reacted. To gauge the focused threshold of the subjects, 10 times of focused inspections were implemented before the training to build an average threshold, and the concentration index threshold was input into the computer algorithm. After these steps, the subjects were instructed to focus on the movement of the ankle on the monitor screen. When the measured concentration index exceeded the threshold of the concentration index where the degree of the subject's concentration was recorded, this information was transferred to the USB output board to activate the FES equipment. However, when the measured concentration index did not exceed the threshold, this information was transferred to the USB output board to turn OFF the FES equipment. Therefore, FES activation occurred based upon the degree of concentration of the subject. In addition, when the measured concentration index exceeded the threshold to turn ON the FES, the electrical stimulation was set to last a maximum of 5 seconds in order to avoid muscle fatigue.

Microstim $^{\circledR}$ (Medel GmBH, Germany) equipment was used for both BCI-FES based training and FES training. This equipment has an adjustable frequency, contraction time, relaxing time, and pulse width and consists of a foot switch, a pair of surface electrodes measuring $50 \times 50 \mathrm{~mm}$, and a stimulator. An inactive electrode was attached to the proximal tibialis anterior (located $5 \mathrm{~cm}$ inferior to the head of fibula), which is an antagonist of plantar flexor muscles, and an active electrode was attached on the distal tibialis anterior (located on the lateral upper $5 \mathrm{~cm}$ of the fibula). The waveform was rectangular biphasic and the therapeutic exercise was adjusted so as not to exceed $50 \mathrm{~mA}$ for the patients to endure as much dorsiflexion as possible. The ramp-up for the maximum intensity was set to take place in 2 seconds, and the duration of stimulation was set to last 7 seconds. In order to avoid muscle fatigue because of electrical stimula- 
Table 2. Comparison of brain waves within groups and between groups $(\mathrm{N}=10)$

\begin{tabular}{|c|c|c|c|c|c|c|}
\hline \multirow{3}{*}{ Parameters } & \multicolumn{4}{|c|}{ Values } & \multicolumn{2}{|c|}{ Change values } \\
\hline & \multicolumn{2}{|c|}{$\begin{array}{l}\text { BCI-FES group } \\
\quad(n=5)\end{array}$} & \multicolumn{2}{|c|}{$\begin{array}{c}\text { FES group } \\
(\mathrm{n}=5)\end{array}$} & \multirow{2}{*}{$\begin{array}{c}\begin{array}{c}\text { BCI-FES group } \\
(\mathrm{n}=5)\end{array} \\
\text { Before-after }\end{array}$} & \multirow{2}{*}{$\begin{array}{c}\begin{array}{c}\text { FES group } \\
(\mathrm{n}=5)\end{array} \\
\text { Before-after }\end{array}$} \\
\hline & Before & After & Before & After & & \\
\hline \multicolumn{7}{|l|}{ Attention index } \\
\hline Fp1 & $0.3271(0.16)$ & $0.6328(0.42)^{*}$ & $0.4530(0.56)$ & $0.5239(0.75)$ & $0.3057(0.34)$ & $0.0709(0.21)$ \\
\hline $\mathrm{Fp} 2$ & $0.2630(0.11)$ & $0.6824(0.49)^{*}$ & $0.2951(0.21)$ & $0.3171(0.28)$ & $0.4194(0.44)$ & $0.0220(0.11)$ \\
\hline \multicolumn{7}{|l|}{ Activation index } \\
\hline Fpl & $1.3375(1.07)$ & $2.8750(2.33)^{*}$ & $1.4625(1.60)$ & $0.9125(0.44)$ & $1.5375(2.23)^{*}$ & $-0.5500(1.22)$ \\
\hline $\mathrm{Fp} 2$ & 2.1375 (1.69) & $2.7950(2.58)$ & $2.3750(1.96)$ & $1.6000(0.77)$ & $0.6575(2.13)$ & $-0.7750(1.88)$ \\
\hline
\end{tabular}

Values are means (SD). ${ }^{*} \mathrm{p}<0.05$ : significant difference within group. $\dagger \mathrm{p}<0.05$ : significant difference between groups

tion, the off-time was set to last 7 seconds, pulse frequency was $35 \mathrm{~Hz}$, and pulse width was $250 \mu \mathrm{s}$.

A Poly-I instrument (Laxtha, Inc., Daejeon, South Korea) was used to take EEG measurements. Each EEG measurement was recorded for one and a half minutes while the subjects were instructed to maintain a comfortable posture with the eyes open and refraining from speaking or moving to minimize interference from artifacts. EEG electrodes were attached to four places on the scalp using the monopolar derivation method. The four sites included frontopolar 1 (Fp1), frontopolar 2 (Fp2), central lobe 3 (C3), and central lobe 4 (C4) in order in accordance with the International 10-20 system. Moreover, reference and ground reference electrodes were placed behind the right earlobe and left earlobe, respectively. The electrodes used were gold-plated disc-shaped EEG electrodes (ElefixZ-401CE, Nihon Kohden, Tokyo, Japan $)^{9)}$.

For EEG data analysis, a quantitative analysis was conducted using Telescan 2.98 (Laxtha Inc., Daejeon, South Korea). Among the overall EEG raw data, 70 seconds of each measurement after excluding the first and last ten seconds was analyzed. Raw EEG data were converted into frequencies using a fast Fourier transformation. Brain waves were categorized following convention into theta $(4-8 \mathrm{~Hz})$, alpha $(8-13 \mathrm{~Hz})$, sensorimotor rhythm $(12-15 \mathrm{~Hz})$, mid beta $(15-20 \mathrm{~Hz})$, and high beta waves $(20-30 \mathrm{~Hz})$. The attention index is the ratio of theta waves to SMR and mid beta waves, and the activation index is the median age frequency $50 \%$.

SPSS version 12.0 was used for all statistical analyses. Nonparametric statistical methods were used due to the small sample size. Wilcoxon's test was used to compare the pretest and posttest results within each group, and the MannWhitney test was performed to compare the two groups before and after training. A p-value $<0.05$ was considered significant.

\section{RESULTS}

Differences in brain activation after the interventions are shown in Table 2. The BCI-FES group showed significant differences in Fp1 and Fp2 of the attention index, and Fp1 of the activation index following the intervention. The FES group showed no significant differences following the intervention. There were significant differences in Fp1 of the activation index between the two groups after the interventions.

\section{DISCUSSION}

Brain waves are defined as electrical signals generated during exchange of information among neurons. They contain important information in a specific pattern varying according to state of awareness and mental activity. High theta wave activity and low beta wave activity are common in patients with attention deficit disorder ${ }^{10)}$. Duffy et al. ${ }^{11)}$ reported that alpha and beta were decreased in quantitative brain wave analysis of elderly individuals, and Kaiser et al. ${ }^{12)}$ reported a significant correlation between event-related desynchronization (ERD) of the unaffected hemisphere with severity of damage in patients recovering from stroke, and between ERD on the affected side and severity of spasticity. Nevertheless, there is very little, research on brain wave analysis in stroke patients. Our study was aimed at grasping the changes in the attention and brain activation indexes depending on the training method applied to stroke patients.

Attention or concentration is defined as the ability to focus and be clearly aware of a stimulus or to react solely to an internal or external stimulus. It is a significantly important psychological task in a variety of settings such as in learning or in sports conditions ${ }^{13)}$. However, inability to concentrate following brain injury is one of the most common problems reported ${ }^{14)}$. Parker et al. ${ }^{15)}$ reported that patients with apoplexy or traumatic brain damage were unable to concentrate on a single problem or situation. Individuals' minds were already distracted before finishing a meaningful task. Duchet et al. ${ }^{16)}$ reported that the deficit of concentration common in stroke or traumatic brain injury can decrease global functioning in learning and daily activities.

Given that each domain in the brain plays a specific role in cognition and specific functions may be impaired if one is affected, the Fp1 domain has been implicated in concentration disorders, whereas judgment and impulse control are relevant to decreased function of the $\mathrm{Fp} 2$ domain $^{17)}$. In our study, the BCI-FES group showed significant differences in the Fp1 and Fp2 attention indices, and the Fp1 activation index. On the other hand, the FES group showed no significant differences. The concentration of the Fp1 and Fp2 domains increased because the chance to exceed the brain wave concentration index threshold increased following training of the patients. In order to measure the degree of concentration during BCI-FES training, electrodes were attached to Fp1 and Fp2 to measure the concentration index of the brain 
wave (SMR+Mid Beta/Theta).

There were a few limitations to this study. First, there were differences between study groups. Even though the threshold of the BCI program was set up based on the concentration index, individuals varied in concentration ability, and the small sample size means that baseline concentration ability differences could significantly alter the results. Second, when the concentration index figure of the BCI program exceeded the threshold to activate the FES, the time of functional electrical stimulation was set up to last $5 \mathrm{sec}$ onds so that muscle fatigue would not occur and to provide a resting phase. However, FES often continuously activated within 5 seconds because of some patients' excellent ability to concentrate, thereby limiting the amount of resting phase. As part of this study, electrodes were attached to Fp 1 and Fp 2 in an isolated environment without external influences to measure the concentration index. However, some electrodes attached to patients who showed a high degree of participation detached from their foreheads due to perspiration. In order to prevent this problem, electrode pads, rather than electrode glue, were used to fix the electrodes, and straps were used to prevent pads from falling off, which may serve as additional sensory stimuli and could potentially bias results. Fourth, attachment of electrodes was studied in a unipolar induction method when measuring brain waves in this study. With the unipolar induction method, electric potential activity can be closely observed, and subcortical depth abnormalities can be easily detected. However, assessing small local differences is difficult, and noise artifacts can limit detection of subtle differences.

In the future, the use of BCI-FES-based training can be revised to rehabilitate not only ankle movements, but also upper extremity motor function. Additional research and clinical use of BCI systems is needed to develop programs suitable for rehabilitation of not only stroke patients but also patients with various disabilities because very little work has been done with these populations.

\section{REFERENCES}

1) Hochberg LR, Serruya MD, Friehs GM, et al.: Neuronal ensemble control of prosthetic devices by a human with tetraplegia. Nature, 2006, 442: 164-171. [Medline] [CrossRef]

2) Birbaumer N, Murguialday AR, Cohen L: Brain-computer interface in paralysis. Curr Opin Neurol, 2008, 21: 634-638. [Medline] [CrossRef]

3) Silvoni $\mathrm{S}$, Ramos-Murguialday $\mathrm{A}$, Cavinato $\mathrm{M}$, et al.: Brain-computer interface in stroke: a review of progress. Clin EEG Neurosci, 2011, 42: 245-252. [Medline] [CrossRef]

4) Kübler A, Birbaumer N: Brain-computer interfaces and communication in paralysis: extinction of goal directed thinking in completely paralysed patients? Clin Neurophysiol, 2008, 119: 2658-2666. [Medline] [CrossRef]

5) Rushton DN: Functional electrical stimulation and rehabilitation-an hypothesis. Med Eng Phys, 2003, 25: 75-78. [Medline] [CrossRef]

6) Granat MH, Ferguson AC, Andrews BJ, et al.: The role of functional electrical stimulation in the rehabilitation of patients with incomplete spinal cord injury—observed benefits during gait studies. Paraplegia, 1993, 31: 207-215. [Medline] [CrossRef]

7) Wassermann E: Changes in motor representation with recovery of motor function after stroke: combined electrophysiological and imaging studies. EEG. Clin Neurophysiol, 1995, 97: S26.

8) Chung EJ, Kim JH, Lee BH: The effects of core stabilization exercise on dynamic balance and gait function in stroke patients. J Phys Ther Sci, 2013, 25: 803-806. [Medline] [CrossRef]

9) Kim JH, Chung EJ, Lee BH: A study of analysis of the brain wave with respected to action observation and motor imagery: a pilot randomized controlled trial. J Phys Ther Sci, 2013, 25: 779-782. [Medline] [CrossRef]

10) Monastra VJ, Lubar JF, Linden M, et al.: Assessing attention deficit hyperactivity disorder via quantitative electroencephalography: an initial validation study. Neuropsychology, 1999, 13: 424-433. [Medline] [CrossRef]

11) Duffy FH, Albert MS, McAnulty G, et al.: Age-related differences in brain electrical activity of healthy subjects. Ann Neurol, 1984, 16: 430-438. [Medline] [CrossRef]

12) Kaiser V, Daly I, Pichiorri F, et al.: Relationship between electrical brain responses to motor imagery and motor impairment in stroke. Stroke, 2012, 43: 2735-2740. [Medline] [CrossRef]

13) Wilson VE, Schmid A, Paper E: Strategies for training concentration. In J. M. Williams (Ed.), Applied sport psychology: Personal growth to peak performance, 5th ed. Mountain View: Mayfield Publishing, 2006, pp 404-422.

14) Cicerone KD: Attention deficits and dual task demands after mild traumatic brain injury. Brain Inj, 1996, 10: 79-90. [Medline] [CrossRef]

15) Parker RS: Traumatic brain injury and neuropsychological impairment. New York: Springer-Verlag, 1990.

16) Duchet J, Christiasen C, Baum C: Cognitive dimensions of peformance in occupational therapy: overcoming human performance deficits. Thorofare, New Jersey: SLACK Inc., 1991.

17) Walker JE, Kozlowski GP, Lawson R: A modular activation/coherence approach to evaluation clinical/QEEG correlations and for guiding neurofeedback training: modular insufficiencies, modular excesses, disconnections, and hyperconnections. J Neurother, 2007, 11: 25-44. [CrossRef] 